\title{
Paciente com Angina e Anomalia Tipo Óstio Único de Artéria Coronária com Origem no Seio de Valsalva Direito
}

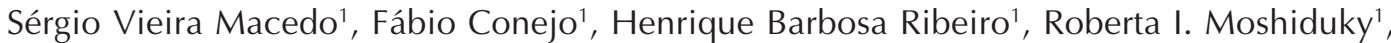 \\ Marco Antonio Perin', Expedito E. Ribeiro ${ }^{1}$
}

\section{RESUMO}

A anomalia cardíaca tipo óstio único de artéria coronária é uma entidade congênita rara, de causa desconhecida. Clinicamente apresenta-se desde a forma assintomática até casos de morte súbita, podendo associar-se a doença arterial coronária aterosclerótica. Apresentamos o caso de uma paciente de 68 anos de idade, com quadro de angina estável desde 2007, que, após três anos de seguimento, em decorrência de piora da angina, foi submetida a cinecoronariografia eletiva. A cateterização seletiva da coronária direita demonstrou anomalia tipo óstio único de artéria coronária, lesão significativa na artéria circunflexa e artéria descendente anterior ocluída no terço médio. A angiotomografia das coronárias demonstrou trajeto benigno da coronária anômala e a paciente evoluiu favoravelmente com o tratamento clínico.

DESCRITORES: Anomalias dos vasos coronários. Doença da artéria coronariana. Seio aórtico.

E $m$ virtude das diferentes definições e classificações, a incidência das anomalias coronárias pode variar, em algumas séries, de $0,1 \%$ a $8,4 \%,{ }^{1}$ dificultando a compreensão de sua real magnitude. Rigatelli et al. ${ }^{2}$ propuseram a subdivisão das anomalias em sete categorias, entre as quais destaca-se a anomalia tipo óstio único de coronária. Essa anomalia está relacionada à apresentação clínica de morte súbita e isquemia coronária, principalmente em adultos jovens. Na literatura, sua incidência é variável, ocorrendo de 0,03\% a $0,4 \%$ dos pacientes submetidos a cateterismo cardíaco. ${ }^{3}$ Relatamos um caso dessa rara anomalia coronária associada a doença aterosclerótica.

1 Instituto do Coração do Hospital das Clínicas da Faculdade de Medicina da Universidade de São Paulo (InCor/HCFMUSP) - São Paulo, SP, Brasil.

Correspondência: Henrique Barbosa Ribeiro. Rua Cônego Eugênio Leite, 866/43 - Cerqueira César - São Paulo, SP, Brasil - CEP 05414-001 E-mail: henrique37@terra.com.br

Recebido em: 22/7/2011 • Aceito em: 17/10/2011

\begin{abstract}
Patient with Angina and Single Coronary Artery Ostium Originating from the Right Sinus of Valsalva

Single coronary artery ostium is a rare congenital disease of unknown origin. Clinical manifestations range from asymptomatic disease to sudden death and it may be associated to atherosclerotic coronary artery disease. We report the case of a 68-year-old woman with stable angina since 2007, who was submitted to elective coronary angiography due to worsening of angina after three years of follow-up. Selective right coronary artery catheterization indicated the presence of single coronary artery ostium, significant lesion of the left circumflex artery, and mid left anterior descending artery occlusion. CT coronary angiography showed a benign course of the anomalous coronary artery and the patient had a favorable outcome with medical treatment.
\end{abstract}

KEY-WORDS: Coronary vessel anomalies. Coronary artery disease. Sinus of Valsalva.

\section{RELATO DO CASO}

Paciente do sexo feminino, com 68 anos de idade, foi admitida no ambulatório do Instituto do Coração do Hospital das Clínicas da Faculdade de Medicina da Universidade de São Paulo (InCor/HCFMUSP - São Paulo, SP), em janeiro de 2007, com queixa de angina aos médios esforços de início recente e melhora com o uso de nitrato sublingual. Apresentava como fatores de risco cardiovascular: hipertensão arterial, dislipidemia e glicemia de jejum alterada, além de pai falecido subitamente aos 40 anos. A estratificação não-invasiva com cintilografia miocárdica demonstrou isquemia moderada anterior e apical. Houve melhora da angina com a otimização das medicações, e a paciente foi acompanhada clinicamente por três anos.

Após esse período, em decorrência de piora da classe funcional de angina, mesmo com o uso regular das medicações, foi solicitada cinecoronariografia, realizada em junho de 2010. Tentou-se a realização de cateterização seletiva, por via femoral, do tronco da 
coronária esquerda no seio esquerdo, sem sucesso (Figura 1A). A cateterização seletiva da artéria coronária direita no seio de Valsalva direito evidenciou a anomalia tipo óstio único de artéria coronária (Figura 1B). Além disso, foi observada lesão significativa na artéria circunflexa, enquanto a artéria descendente anterior en- contrava-se ocluída em seu terço médio (Figura 1B). Angiotomografia coronária de 320 detectores (angioTC-320) confirmou esses achados e demonstrou que o trajeto da coronária anômala era anterior à artéria pulmonar (Figura 2). Tendo em vista a melhora dos sintomas com um novo ajuste do tratamento clínico, optou-se por manter

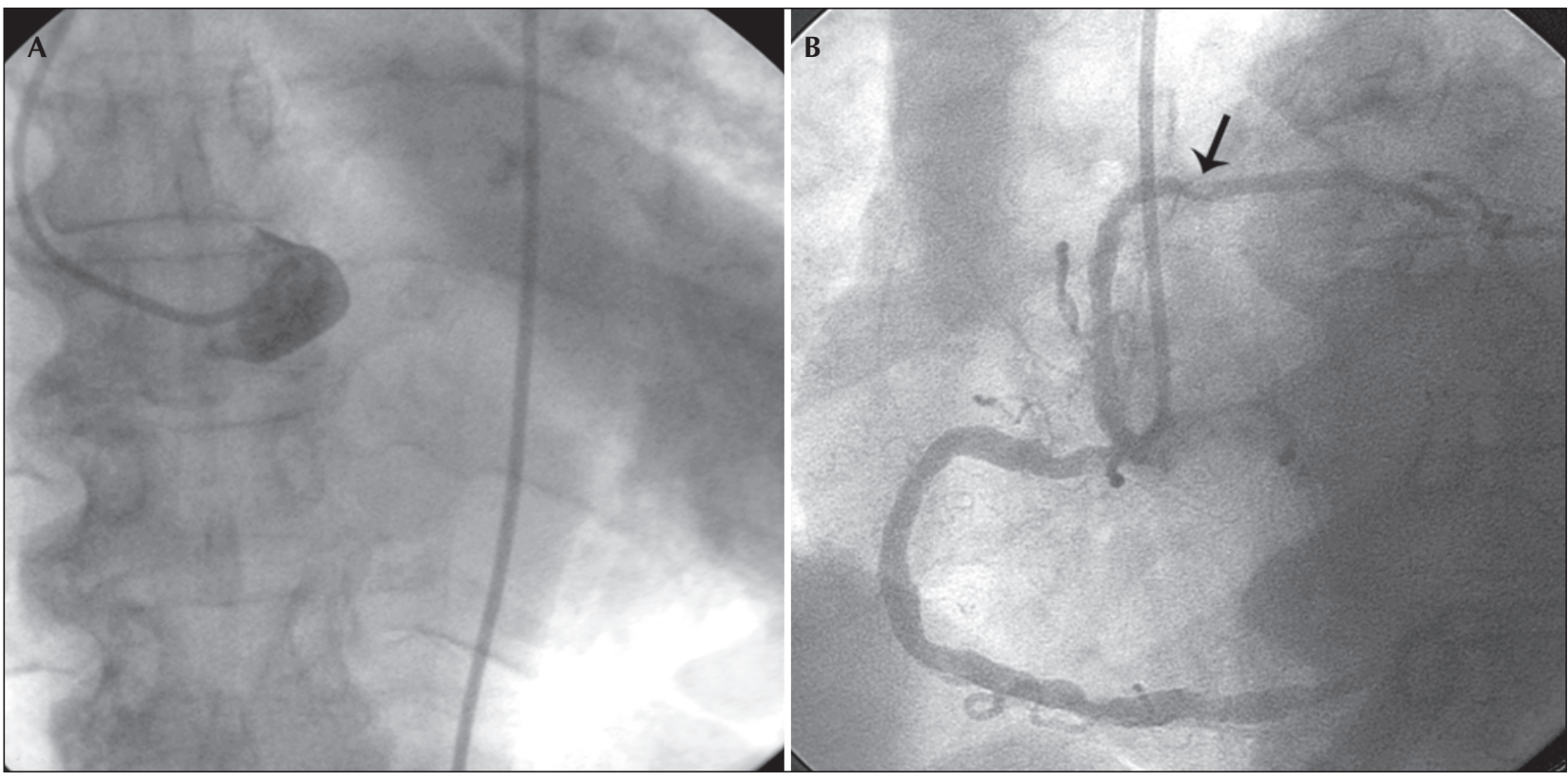

Figura 1 - Em A, cinecoronariografia evidenciando a não-opacificação do tronco da coronária esquerda no seio esquerdo. Em B, cinecoronariografia em projeção oblíqua anterior esquerda, demonstrando lesão significativa na artéria circunflexa (seta).
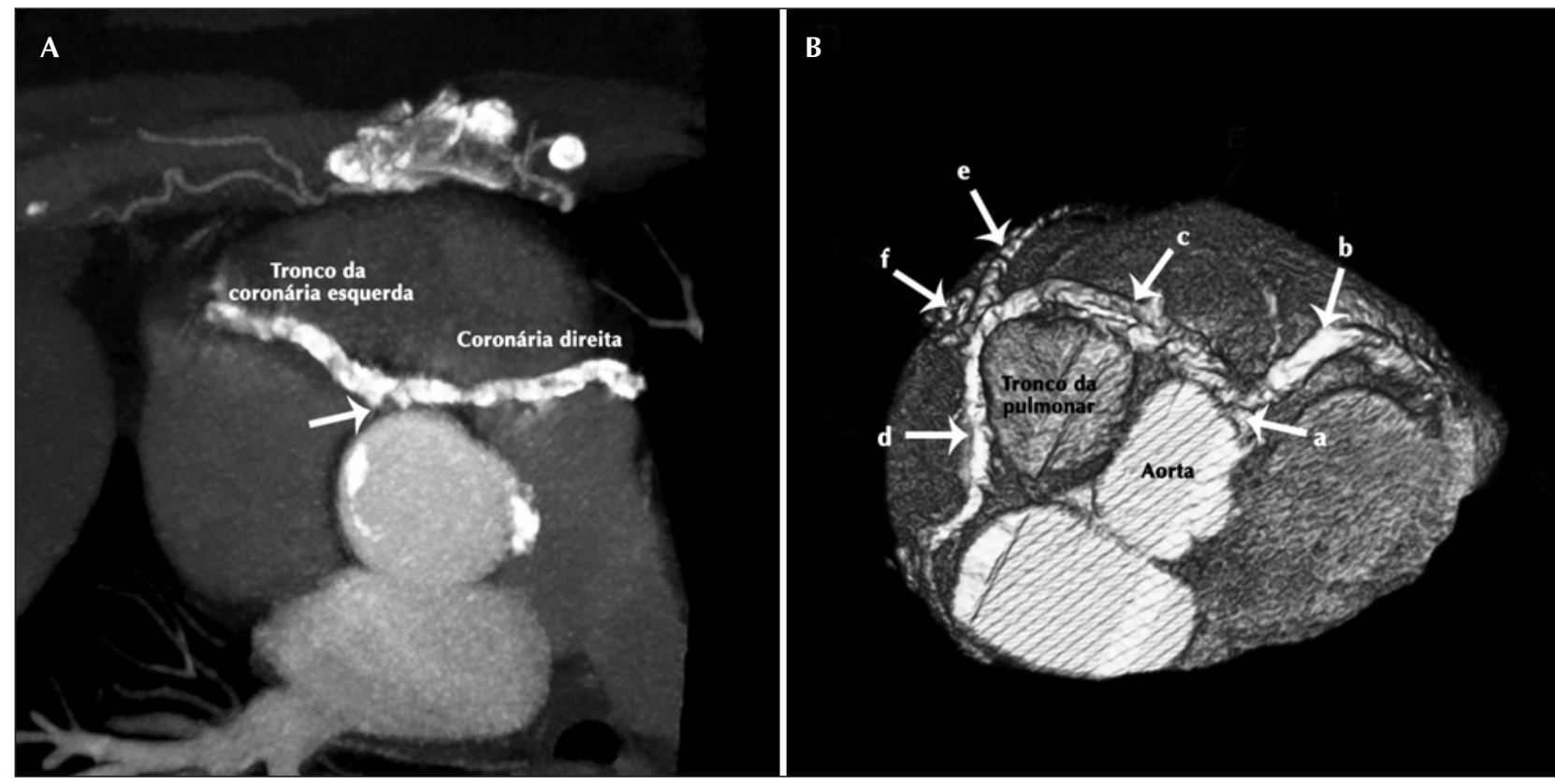

Figura 2 - Em A, corte axial de angiotomografia coronária de 320 detectores confirmando a presença de tronco único emergindo do seio direito (seta). Em B, angiotomografia coronária de 320 detectores com reconstrução tridimensional, vista pela base do coração (a, tronco coronário único saindo do seio direito; b, artéria coronária direita; c, tronco da coronária esquerda; d, artéria circunflexa; e, artéria descendente anterior; $\mathrm{f}$, ramo intermédio). 
a paciente clinicamente, sem angioplastia ou cirurgia. Na evolução de um ano, a paciente mantém-se assintomática.

\section{DISCUSSÃO}

O primeiro relato de anomalia tipo óstio único de artéria coronária foi feito por $\mathrm{Hyrtl}^{4}$, em 1841, sendo essa anomalia considerada um dos sete subtipos, segundo a classificação de Rigatelli et al. ${ }^{2}$. Pode se associar a morte súbita e angina, principalmente quando assume trajeto interarterial (entre a artéria pulmonar e a aorta), apresentando sua maior letalidade em adultos jovens, diferente do caso relatado, em que o trajeto era anterior à artéria pulmonar. A maior parte dos pacientes com origem do tronco da coronária esquerda do seio contralateral (cerca de 59\%) morre antes dos 20 anos de idade, em geral durante ou logo após exercício físico vigoroso ( $81 \%$ dos casos). ${ }^{5}$ Burke et al. ${ }^{6}$ relataram que, entre indivíduos de 14 anos a 40 anos, as anomalias de artérias coronárias estavam presentes em $12 \%$ das mortes relacionadas à prática esportiva, enquanto apenas $1,2 \%$ das mortes não relacionadas à atividade física estavam associadas às anomalias.

A patogênese da anomalia tipo óstio único de artéria coronária ainda é desconhecida e sua relação familiar é incerta, já que existem poucos relatos até hoje da associação familiar dessa doença. Laureti et al. ${ }^{7}$ relataram o caso de dois irmãos que possuíam origem anômala do tronco da coronária esquerda do seio contralateral, porém em um deles o trajeto era interarterial e no outro, retroaórtico. Essa variação de origem entre as anomalias tipo óstio único de artéria coronária de dois familiares pode ter ocorrido no caso aqui relatado; contudo, como o pai da paciente em questão faleceu subitamente e não foi submetido a cinecoronariografia, isso não pode ser confirmado.

O espectro da apresentação clínica é bastante variável, podendo se apresentar como angina, síncope, dispneia, arritmias ventriculares, insuficiência cardíaca ou morte súbita. Há várias teorias para explicar a indução de isquemia nessa afecção, porém nenhuma totalmente demonstrada. A primeira delas seria a grande angulação que a artéria anômala desenvolve ao se originar da aorta e se curvar para seguir trajeto em direção ao seio contralateral. Isso torna o óstio menor e facilmente compressível pela aorta durante o exercício. ${ }^{1}$ A segunda, e talvez a mais aceita, aconteceria apenas quando o vaso percorre trajeto entre a artéria pulmonar e a aorta, sofrendo compressão extrínseca dos mesmos, especialmente durante atividade física, quando há natural expansão desses vasos. Outra hipótese seria a disfunção endotelial gerada pelo trajeto anômalo, induzindo espasmos coronários. ${ }^{1,5,6}$

Quando houver suspeita clínica, é importante, principalmente em atletas, fazer a investigação comple- mentar com teste ergométrico, ecocardiograma, angiotomografia das coronárias e/ou cinecoronariografia, esta última indicada, principalmente, naqueles com suspeita de doença aterosclerótica associada, quando a investigação não-invasiva não foi suficiente para descartar esse diagnóstico. O trajeto da artéria coronária também deve ser avaliado, sendo esse um dos pilares para a definição prognóstica e terapêutica do paciente. O óstio único de artéria coronária pode seguir quatro trajetos: retroaórtico, septal, anterior e interarterial, sendo este último associado a apresentação clínica maligna, devendo, na maior parte das vezes, receber tratamento cirúrgico, com reimplante ou até mesmo ligadura das coronárias, seguida de enxerto de veia safena ou artéria torácica interna.

No caso em questão, a paciente apresentava trajeto anômalo benigno e os sintomas justificavam-se pela doença aterosclerótica coronária. Nesses casos, o paciente pode ser submetido a revascularização cirúrgica do miocárdio, tratamento clínico isolado ou tratamento percutâneo, de acordo com a indicação clínica, independentemente da anomalia, o que já foi feito por outros autores, com sucesso, na literatura. ${ }^{8,9}$

\section{CONFLITO DE INTERESSES}

Os autores declaram não haver conflito de interesses relacionado a este manuscrito.

\section{REFERÊNCIAS}

1. Barriales-Villa R, Morís de la Tassa C. Congenital coronary artery anomalies with origin in the contralateral sinus of Valsalva: which approach should we take? Rev Esp Cardiol. 2006;59(4):360-70

2. Rigatelli G, Docali G, Rossi P, Bovolon D, Rossi D, Bandello $A$, et al. Congenital coronary artery anomalies angiographic classification revisited. Int J Cardiovasc Imaging. 2003;19(5): 361-6.

3. Stauffer JC, Sigwart U, Vogt P, Aymon J, Kappenberger L. Transluminal angioplasty of a single coronary artery. Am Heart J. 1991;122(2):569-71.

4. Hyrtl J. Einige in chirurgischer hinsicht wichtige gefässvarietäten. Med Jahrb Österr Staats. 1841;33:17-38.

5. Angelini P, Velasco JA, Flamm S. Coronary anomalies: incidence, pathophysiology, and clinical relevance. Circulation. 2002; 105(20):2449-54.

6. Burke AP, Farb A, Virmani R, Goodin J, Smialek JE. Sportsrelated and non-sports-related sudden cardiac death in young adults. Am Heart J. 1991;121(2 Pt 1):568-75.

7. Laureti JM, Singh K, Blankenship J. Anomalous coronary arteries: a familial clustering. Clin Cardiol. 2005;28(10):488-90.

8. Angelini P. Coronary artery anomalies: an entity in search of an identity. Circulation. 2007;115(10):1296-305.

9. Yokoyama HA, Pessoa CM, Carvalho FC, Campos RF, Franco RJ, Bregagnollo E. Intervenção coronária percutânea em artéria coronária única em paciente com angina instável de alto risco. Arq Bras Cardiol. 2007;88(3):e53-5. 